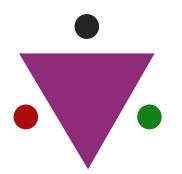

IJCRR

Section: Healthcare

Sci. Journal Impact

Factor: 6.1 (2018)

ICV: 90.90 (2018)

(c) (i) (9)

Copyright@IJCRR

\title{
Prophylactic Measures to be Taken by Oral Health Care Professionals During a Pandemic Outbreak of COVID-19
}

\author{
Avula Kishore Kumar ${ }^{1}$, Aluru Sudheer ${ }^{2}$, Gujjula Sravanthi ${ }^{3}$, \\ Laxmi Tejaswin Polepalle ${ }^{4}$, Nagireddy Ravindra Reddy ${ }^{1}$, Firoz babu P $^{5}$, \\ Mannur Ismail Shaik ${ }^{6}$, Narasimha Golla ${ }^{7}$
}

\begin{abstract}
'Department of Periodontics and Implantology, CKS Theja Institute of Dental Science and Research Centre, Andhra Pradesh, India; ${ }^{2}$ Diviion of Animal Biotechnology, Department of Zoology, Sri Venkateswara University, Tirupati-517502, Andhra Pradesh, India; ${ }^{3}$ Department of Prosthodontics and Implantology, CKS Theja Institute of Dental Science and Research Centre, Andhra Pradesh, India; ${ }^{4 D}$ epartment of Periodontics and Implantology, SIBAR Institute of Dental Science and Research Centre, Andhra Pradesh, India; ${ }^{5}$ Department of Orthodontics, CKS Theja Institute of Dental Science and Research Centre, Andhra Pradesh, India; ${ }^{6}$ Faculty of Fisheries and Food Science, Universiti Malaysia Terengganu, 21030 Kuala Nerus, Terengganu, Malaysia; ${ }^{7}$ Applied Microbiology Laboratory, Department of Virology, S.V.U. College of Sciences, Sri Venkateswara University, Tirupati, 517502, Andhra Pradesh, India.
\end{abstract}

\section{ABSTRACT}

Many viruses have been posing threat to the world from time to time and recently in this category, a novel coronavirus called COVID-19 (SARS-CoV2) was detected in December 2019 and is causing havoc all over the world. COVID-19 spreading through person-to-person transmission routes including direct transmission, like cough, sneeze, droplet inhalation and contact transmission, like contact with oral, nasal and eye mucous membranes. The operators in oral health care profession expose to great risk of COVID-19 infection due to face-to-face communication and their exposure to fluids from the conjunctiva, oral cavity, nose and also other body fluids, and handling with the sharp instruments. Oral health care professionals (dentists and dental hygienists) play a prime role in preventing the spread of COVID-19. Several dental hospitals and clinics have been completely closed or have been providing minimal treatment for emergency cases. However, prophylactic and emergency treatments are being provided in some countries and a few supporting regular dental treatment. Oral health practitioners are prone to risk as they are hardly prepared for such unexpected global outbreak. Lack of universal protocol or guidelines to control the infection and dental care provision during such a pandemic condition could be of better help. The present study fills in the missing gap with necessary recommendations comprising of preventive steps for disease/infection transmission during dental practice to block the operatorto-patient and vice-versa transmission routes in dental clinics and hospitals.

Key Words: COVID-19, Coronavirus, Dental, Precautions, Lockdown, Awareness

\section{INTRODUCTION}

Viruses have always been challenging to mankind with their dynamicity in structure, virulence, prevalence, transmission, and potentiality to claim lives. One among those recently discovered is the Novel Coronavirus 2019. In late December 2019, Wuhan City province of China suffered through a pneumonia outbreak, which kept spreading at an exponential rate to other parts of China and neighbouring countries. Even before it became clear about its aetiology and mode of transmission, it was reported in more than 25 other countries and WHO declared it as a Public Health
Emergency of International Concern on January 30, 2020. Within no time, Covid-19 had spread around 18 countries in which four countries reported human-to-human transmission. On February 26, 2020, the first case of this disease was recorded in the United States, which was not imported from China. As of today, April 10, 2020, COVID-19 has affected around 210 countries and territories all over the globe including, two international conveyances namely, Diamond Princess Cruise harboured at Yokohama, Japan, and Holland America's MS Zaandam Cruise as retrieved from WHO coronavirus disease situation dashboard on April 11, 2020.2,3

\section{Corresponding Author:}

Dr. N. Ravindra Reddy, Professor and HOD, Department of Periodontics and Implantology, CKS Theja Institute of Dental Sciences and Research, Tirupathi, Andhra Pradesh 517501; Ph: 9618543243; Email: cksperio@gmail.com

ISSN: 2231-2196 (Print)

Received: 10.08.2020
ISSN: 0975-5241 (Online)

Revised: 19.09 .2020
Accepted: 22.11 .2020 
DISTINCTIVE FEATURES OF NOVEL CORONAVIRUS (COVID-19)

The causative organism for COVID-19 was identified as the seventh member of the coronaviruses family Coronaviridae of the order Nidovirales, possessing huge single-stranded positive RNA (+ssRNA) as genetic material. It belongs to one of the four coronavirus genera's, namely, $\alpha, \beta, \gamma$, and $\delta$ which can cause the infectious diseases among human and vertebrates, of which, $\alpha$ and $\beta$ mostly infect the pulmonary, central nervous system (CNS) and gastrointestinal (GI) tract of human and mammals, while $\gamma$ and $\delta$ primarily infect the birds. ${ }^{4}$

\section{Aetiology}

This virus is also speculated to have an animal origin with the capacity of the cross-species barrier to reach humans, similar to MERS and SARS of which SARS is believed to be originated from bats and then pass on to Himalayan palm civet, while MERS-CoV might have moved on from dromedary camel to human. Chan et al. in 2019 have proven that the genome of the human $\mathrm{CoVs}(\mathrm{HCoV})$, isolated from Wuhan returned atypical pneumonia cluster patient had 89\% similarity with bat SARS-CoV and $82 \%$ identity with human SARS-CoV. ${ }^{5}$ However, it is of no certainty that a possible intermediate host may exist as a potential intermediate between human and bat is identified, and hence it could be as a result of a possible mutation in original strain.

\section{Transmission}

Initially, COVID-19 was believed to be animal to the human transmission when it was first reported in Wuhan's Huanan Seafood Wholesale Market. As there were no subsequent patients linked with exposure, it was ruled out, and human to human transmission was noticed as the route of transmission and spread of COVID-19. The possibility of asymptomatic individuals acting as carriers in transmitting the virus is observed and from the data, isolation is suggested to be the ideal way to restrict this epidemic.

The transmission is believed to occur through aerosols, which arise from respiratory droplets during sneezing or coughing. There is every possible chance of elevated aerosols due to lengthier exposure in closed spaces such as COVID-19 isolation wards. According to recent observations, the close contact between individuals is found to be necessary for transmission and hence, it is primarily limited to the family members, doctors, health care professionals, workers, and all other close contacts.

Reports out of China revealed that, the same means of transmission between close contacts and persons who attended the same social gatherings, or confined to one place such as office spaces or cruise ships or been a part of a religious gathering. ${ }^{6}$ This poses a serious threat for community trans- mission if left uncontained as experienced by India, where a large mass India experienced a similar incident where (Nizamuddin incident) around 2,000 people were found to be staying at Markaz Nizamuddin, New Delhi during the outbreak. Some of whom being international tourists have been asymptomatic carriers or being in latent phase might have transmitted to fellow members who later on dispersed to their native places all over India. This incident was one of the major transmissions witnessed by India.

\section{The incubation period of COVID-19}

The incubation period is the time interval between the moment of exposure to an infectious state until the signs and symptoms of the disease appear. An estimated time of 2 to 14 days of latency is reported by $\mathrm{WHO}^{7}$ and $\mathrm{CDC}^{8}{ }^{8}$ While China's National Health Commission (NHC) reported 10 to 14 days of window period. ${ }^{9}$ However, a case with 27 days latency period at Hubei Province ${ }^{10}$ and a study with 19 days latency was recorded in February, 2020. ${ }^{11}$

\section{Symptoms}

Dry Cough, Fever and Fatigue were the common symptoms experienced by most of the infective persons and very few among those had difficulty in breathing, abnormal chest radiographs with invasive lesions of both lungs. Huang et al. 2020 reported that diarrhoea, headache, sputum production and hemoptysis also found in several cases. ${ }^{12}$

Angiotensin-converting enzyme 2 (ACE-2) is a cell surface receptor which is present on multiple human organs. ${ }^{13}$ As it is the functional receptor being the site for COVID-19 virus, it leads to dysfunction in multiple organs in addition to respiratory failure unlike other respiratory diseases such as the Middle East Respiratory Syndrome Coronavirus (MERS-CoV, 2012) and Severe Acute Respiratory System Coronavirus (SARS-CoV, 2002-2003). ${ }^{14,15}$

\section{DENTAL PERSPECTIVE}

All health care professionals, including doctors, surgeons, helpers and support staff are at greater risk of disease contraction from patients as it is nearly impossible to identify COVID-19 positives from superficial observation as few may be asymptomatic being in the latent phase. Hence it is mandated to follow statutory precautions while handling patients as per the Center for Disease Control guidelines.

\section{Possible Transmission Routes of Covid-19 in Dental Clinics}

Oral health care professionals and their patients are often at risk of exposure to virulent microorganisms, including bacteria and viruses that infect the oral cavity as well as the respiratory tract. Dental procedures always posses the threat of 
COVID-19 transmission as it involves direct interaction with patients, and also their saliva, blood and other body fluids.

\section{Airborne spread}

The airborne spread of COVID-19 is a known fact. The literature reveals that, the possible contamination of droplets and aerosols produced during dental procedures with virus. ${ }^{16}$ Hence, the transmission of COVID-19 becomes a major concern as it's next to impossible to avoid the patient's saliva and blood combined aerosols and droplets in dental setups during their procedures. ${ }^{17}$

\section{Discipline-wise safety measures to curb aero- sol transmission}

Endodontics should take extra precautions during root canal treatment to minimize hand contact with inanimate surfaces around the clinic and should practice usage of a rubber dam during the procedures. Oral-maxillofacial surgeons should perform on patient-facing up in a supine posture to keep away from the patient's breath. Periodontists should preferably use manual scalers over mechanical to minimize aerosols. However, the usage of ultrasonic instruments with vacuum pumps can also be more effective only when combined with personal protective gear.

Restorative and pediatric dentists in typical cases should practice either non-traumatic restoratives or chemo-chemical removal techniques. They are also advised to restrict the usage of rotary appliances and under obligatory situations; the practitioner might isolate the areas with rubber dams. Prosthodontists could be more prone to contagion. Hence timely usage of suction pumps and usage of aptly sized impression trays along with application oral mucosal anaesthesia before the procedure is highly recommended. For fixed partial dentures $\mathrm{Li}$ et. al., $2004^{18}$ recommends modification treatment strategy either by the usage of split rubber dam or designing supra-gingival margin for the posterior bridge can be implemented in current situations. While for a removable partial denture or complete denture the practitioner should avoid maximum contact with inanimate surfaces after contacting the patient's saliva. Similarly, all the instruments which were used for a patient need to be thoroughly disinfected immediately without further delay.

\section{Spread by Contact}

Oral health care or dental professional is prone to direct or indirect contact with the human fluids, including saliva and Gingival Crevicular Fluid (GCF), patient drapes, and used clinical instruments or surfaces which makes a possible route for virus transmission. In addition to that, contact with conjunctival, nasal or oral mucosa along with propelling droplets and aerosols bearing the virus from an infected patient during a dental procedure or by cough or talk poses a high risk for a dentist. Hence, stringent measures need to be taken to contain the contact spread of COVID-19.

\section{Contaminated Surfaces Spread}

Human coronaviruses like SARS-CoV, MERS-CoV, or $\mathrm{HCoV}$ can persist on various surfaces like metal, plastic or glass for up to few of days. ${ }^{19,20}$ Therefore, those contaminated surfaces frequently contacted in healthcare settings which are a possible source for transmission of COVID-19.

\section{Infection Controls at Dental Practices}

Dental professionals should be conversant that, how COVID-19 spread, the way to recognize COVID-19 infected patients and what are the additional protective measures should be adopted during the dental practice, to stop the COVID-19 transmission. In this study, we recommend the COVID-19 infection control measures that ought to be followed by dental professionals because of aerosols and droplets were considered to be main spread routes of COVID-19.

\section{INDIVIDUAL DEFENSIVE MEASURES FOR THE DENTAL EXPERTS}

In light of the chance of the spread of COVID-19, three-level defensive proportions of the dental experts are suggested for explicit circumstances. ${ }^{1}$ First level security (standard insurance for staff in clinical settings). Usage of a protective face shield or eye goggles, disposable latex or Nitrile gloves along with wearing an expendable working top, dispensable careful cover, and working garments (white coat) as needed. ${ }^{2}$ Second level care (propelled security for dental experts). Wearing dispensable specialist top, expendable careful cover covering mouth, protective eye goggles, face shield and working garments (apron) along with an expendable detachment dress or careful garments outside and expendable latex gloves. ${ }^{3}$ Third level protection (extra safety measures when possible contact with doubtful or affirmed COVID-19 patient). Albeit a patient with COVID-19 disease isn't relied upon to be attended at a dental facility, yet there could be an improbable occasion, which this could happen, and the dentist can't evade close contact. Instead, he/she needs an extraordinary defensive to outwear. If defensive outwear isn't accessible, working garments (white coat) with additional expendable defensive outside garments such as impervious shoe covers, face shields, protective goggles, disposable gloves, disposable top and a veil are to be worn.

\section{Mouthrinse Before Dental Methods}

It is widely accepted that a preoperational rise with an antimicrobial mouthwash will decrease most the number of oral organisms. Most of these mouth rinses contain chlorhexidine as an active ingredient which is considered to be ineffective against COVID-19. But as it's aware that COVID-19 is helpless against oxidation, a pre-procedural mouthwash with oxidative specialists, for example, $1 \%$ hydrogen peroxide or $0.2 \%$ povidone is suggested, to diminish the salivary heap 
of oral microorganisms, including potential COVID-19 carriage. A pre-procedural mouth rinse would be generally valuable in situations when an elastic dam can't be utilized.

\section{Rubber Dam Segregation}

The utilization of elastic dams can essentially limit the creation of salivation and blood-sullied vaporized or splash, especially in situations when rapid air-rotor Handpieces, dental ultrasonic and piezoelectric ultrasonic scalers are utilized. Utilization of rubber dams could essentially diminish airborne particles or aerosols in the 1-meter measurement of the operational field by $70 \% .{ }^{21}$ Additional high volume suction for vaporized and scatter needs to be utilized alongside normal suction when the rubber dam is applied. ${ }^{22}$ Right now, the usage of total four-hand activity is additionally fundamental. On the off chance that elastic dam disconnection is absurd at times, manual gadgets are to be opted to limit the spread of airborne infections. For instance, periodontal scaling and caries expulsion can be done using a hand scaler and caries excavator.

\section{Anti-retraction Handpiece}

During dental procedures, debris and fluids may be expelled during aspiration done with air-rotor dental handpiece without anti retraction valves. Infectious microbes can infiltrate air and contaminate water tubes of a dental unit leading to cross-contamination. According to $\mathrm{Hu}$ et al. $\left({ }^{2007) 23}\right.$, the backflow of oral bacteria into tubes of dental setup is significantly less likely when an anti-retraction high-speed dental handpiece is used instead of handpiece without anti-retraction and hence, their utilization need to be highly discouraged during this COVID-19 pandemic. ${ }^{23}$ Hence, we are of the strong opinion that usage of specially designed valves containing anti-reflux equipment or anti-retraction dental Handpieces with valves shall be an efficient preventive step against cross-infection during pandemics. ${ }^{22}$

\section{Sterilization of the Dental Hospitals/Work Places/Clinics}

Stringent sanitization measures have to be adopted by healthcare setups where people have the possibility of coming in contact with surfaces such as appliances, staircase railings, door handles, furniture like chairs, desks, elevator operation panel and any further. They need to follow the protocol of Management of Surface Cleaning and Disinfection of Medical Environment (WS/T 512-2016) released by the National Health Commission of the People's Republic of China on December 27, 2016. People should be intimated to wear a mask and avoid direct contact with button panel and other surfaces in elevators despite it being cleaned regularly with disinfectants. Table 1 , represents measures to contain nosocomial transmission of COVID 19 in India.

\section{Medical Waste Management}

Medical waste contains infectious or potentially infectious material. It has to be timely transported to a temporary storage unit inside the premises of the medical institute or hospital. The domestic and medical waste generated from treating COVID-19 patients or suspects thereof hereafter referred to as infectious medical waste needs to be packed in a yellow coloured double-layer medical waste package bags and secured with a gooseneck knot. These should be properly labelled and disposed of as per institutional norms of medical waste disposal. However, dental instruments and tools which are reusable need to be properly stored under the Protocol for the Disinfection and Sterilization of Dental Instrument (WS 506-2016) released by the National Health Commission of the People's Republic of China after through pretreatment, cleaning and sterilization.

Table 1: Measures to contain nosocomial transmission of COVID 19 in India.

\begin{tabular}{|c|c|c|c|}
\hline Preventive aids & $\begin{array}{l}\text { Procedures generating } \\
\text { aerosols }\end{array}$ & $\begin{array}{l}\text { Clinical wards and spaces with } \\
\text { constant people exposure }\end{array}$ & $\begin{array}{l}\text { Clinical wards and spaces } \\
\text { without constant people } \\
\text { exposure }\end{array}$ \\
\hline Hand sanitation & Needed & Needed & Needed \\
\hline Type of Face mask & N95 respiratory mask & Procedural mask / Surgical mask & $\begin{array}{l}\text { Procedural mask / Surgical } \\
\text { mask }\end{array}$ \\
\hline $\begin{array}{l}\text { Full back protective gown/ } \\
\text { drape }\end{array}$ & Level 1 or 3 as per AAMIc & \multirow{4}{*}{$\begin{array}{l}\text { Statutory hygiene precautions } \\
\text { along with disease transmission- } \\
\text { based precautions as per need. }\end{array}$} & Not Needed \\
\hline $\begin{array}{l}\text { Examination gloves - Dispos- } \\
\text { able }\end{array}$ & Needed & & Not Needed \\
\hline Safety eyewear & $\begin{array}{l}\text { Protective face shield/ den- } \\
\text { tal goggles }\end{array}$ & & Not Needed \\
\hline Bouffant cap & Optional & & Not Needed \\
\hline
\end{tabular}


a) Procedures such as nebulizer therapy, open suction of respiratory tract, bronchoscopy, intubation, high-frequency oscillatory ventilation, non-invasive positive pressure ventilation, cardiopulmonary sputum induction.

b) Front office, outpatient wards, procedure rooms, radiology wings, waiting for spaces.

c) Protective clothing against liquid contamination by AMMI (Association for the Advancement of Medical Instrumentation PB70:2003) guidelines suggest Level 1 drape is used in anticipation of protection against the less amount of liquid exposure and Level 3 is used where the possibility of liquid exposure is more in quantity (Table 1).

\section{Precautions to be taken by dental profession- als after the lockdown ends}

- If possible, don't carry out aerosol related treatments (Airrotor and Scaler) till End of May-2020.

- Strict Written informed consent, screening, and Undertaking from all patients regarding COVID and other diseases in general.

- Before and after each patient's observation, patient's dental chair, hand rests, spittoon, tray, buttons, headlight switch and door handles to be disinfected with alcohol scrub.

- Ask the patient to keep their hands in their pockets and without touching anywhere to sit on the Dental Chair.

- All asymptomatic treatments to be deferred.

- Proper Donning and Doffing protocols if using PPE.

- Any procedures generating splatter to be done with complete Disposable PPE for all operating Doctors and Assistants along with Rubber Dam isolation and preferably appointment kept at the end of the day.

- Post splatters treatment, strict fumigation of Operatory need to be performed.

- Routine procedures like OPD, Crown cementation, ART, can be carried out.

- The water reservoir of the chair be added $\mathrm{NaOCl} 2.5 \%$ or $\mathrm{H}_{2} \mathrm{O}_{2} 0.5 \%$

- Betadine throat gargles, just before any treatment is to be initiated.

- Minimal follow-ups.

- Dispose of the PPEs and Biomedical waste judiciously.

- Fees to be encouraged to be paid by Digital routes

- Before and after each session, meticulous floor mopping, handle disinfection, Dental chair, X-rays, Trays, Spittoon, Operatory, and waiting area needs to be implemented.

- It is highly recommended to use single-use/disposable under dams and root canal files. Other regular instruments used for diagnosis or treatment need to be sealed and autoclaved. Soaking in Orocid like solutions before autoclaving is advised for used burs.

- Scrubbing of a handpiece with a disinfectant before and after ever-patient should be practised without fail.
- Intra procedure, hi-vac suction used in conjunction with saliva ejectors, should be used to minimize aerosol dissemination.

- Rubber dams must be made mandatory, along with adequate training on its usage will form the best barrier to prevent aerosol formation.

- Impressions should be effectively disinfected before dispatch to laboratories.

- Fumigation with a quaternary ammonium compound must be performed every day, to ensure that all low contact areas are also disinfected.

- Minimal use of Air Conditioners and restrict its use during Aerosol generating procedures. Also, regular cleaning of its filters and during Fumigation/Fogging should be on as the fumigant reaches the filters throughout.

- Waste disposal must stringently follow biomedical waste disposal protocols as defined by the Municipal authorities.

- Please convey beforehand or better put up a notice that due to added precautions, PPEs, etc. being taken, resulting in added costs, treatment for each procedure would increase by 10 to $15 \%$ minimum.

- Educating people with facts enlightens them out of unnecessary fears. As a responsible doctor, you need to give them the confidence by emphasizing the facts regarding the spread and necessary precautions.

- Adhering to all these practices creates a safe environment for patients and treatment providers. It creates a note of confidence among your patients seeking your service.

\section{THOUGHTS FOR FUTURE}

COVID-19 outbreak had unveiled the empty spaces in public health care internationally. Dentistry too failed to face emergencies of COVID19. This has to be considered as a learning opportunity to identify the role of the oral health care professional during health emergencies to improvise and implement better patient service along with personal protection from contagion. With serial outbreaks of SARS, MERS, Ebola, H1N1 and COVID19, it is evident that these emergencies are not rare anymore and we need to be prepared to fight them actively. Profound research into public dental health, basic dental education at all levels and appropriate clinical practices have to be implemented for a better future.

Inclusion of obligatory use of surgical masks, single-use appliances, tools, masks, gowns and other dental tools into Dental curriculum will help in the continuation of these practices during regular practice at their later stage.

Usage of chair-side rapid testing kits to identify a particular salivary sample for possible microbial contamination at the molecular level is an advisable way to early detect and protect against any communicable microbial infection, even at 
an asymptomatic stage. Further to identification, back-tracing the contacts can help in disease containment. These tests will also help us to identify the people who have developed pooled immunity due to infection, who can be used to extract readymade antibodies during emergencies.

Aerosol spread posses the highest threat in dental procedures for droplet borne infections like COVID-19. In such cases, negative pressure rooms prove to be most effective, which are generally used to control respiratory diseases. Dental professionals around the world need to consider this as a viable option for the future and has to be brought into practice at learning centres and government facilities.

\section{CONCLUSION}

Although there exists a lockdown due to epidemic, few patients still rush to emergency dental facilities and hospitals for treatment. As novel coronavirus COVID-19 is an airborne disease and can be spread via contact with contaminated surfaces, we have suggested all possible methods to contain the spread at dental clinics. We have summarized about dental procedures to be followed during diagnosis, treatment, patient evaluation, personal hygiene, equipment sanitization and protective measures for dental professionals, the right kind of equipment to be used and medical waste management as part of preventing the transmission of COVID19 at dental clinics. Fortunately, this virus is sensitive to regular disinfectants used in day to day dentistry procedures and by following stringent sanitization procedures as discussed will reduce the risk for patients and dentists. A dental professional or team will rarely get to face a COVID19 case, but if any latent or carrier visits the facility, he/ she poses a significant transmission risk to the entire team. Hence, knowledge and awareness among dental professionals are of vital importance against the virus. Its dark times for dentistry. With warnings from $\mathrm{WHO}$ about long-lasting persistence of COVID1924, it has become a nightmare for dentists and oral care professionals out there. However, life can't be contained. It has to move on. Clinics can't be shut down forever. So, with appropriate knowledge and prophylactic measures discussed above, it is viable for the reopening of dental practices globally.

\section{ACKNOWLEDGEMENT}

Authors acknowledge the immense support from CKS Theja Dental College, Tirupati, Andhra Pradesh, India and thank the scholars whose articles are cited and included in references of this manuscript. The authors are also grateful to authors/editors / publishers of all those articles, journals and books from where the literature for this article has been reviewed and discussed.

\section{Conflict of Interest and Funding Information}

Authors declare no conflict of interest and state no receive any funding from any agency or institution.

\section{Source of Funding: Nil}

\section{REFERENCES}

1. Wang C, Horby PW, Hayden FG, Gao GF. A novel coronavirus outbreak of global health concern. Lancet 2020;395(10223):47073.

2. Worldometer. COVID-19 Coronavirus Pandemic data. https:// www.worldometers.info/coronavirus/. Accessed 11/04/2020.

3. World Health Organization. https://who.sprinklr.com/explorer. Accessed 11/04/2020.

4. Fehr AR, Perlman S. Coronaviruses: an overview of their replication and pathogenesis. Meth Mol Biol 2015;1282:1-23.

5. Chan JF, Kok KH, Zhu Z, Chu H, To KK, Yuan S, Yuen KY. Genomic characterization of the 2019 novel human-pathogenic coronavirus isolated from a patient with atypical pneumonia after visiting Wuhan. Emerg Microb Infect 2020;9(1):221-36.

6. Juliet B, Delia E, Johan G, David LH, Chikwe I, Gary K, et al. COVID-19: towards controlling of a pandemic. Lancet 2020;395(10229):1015-8.

7. World Health Organization (WHO). Novel Coronavirus (2019nCoV) Situation Report-7 - February 11, 2020. https:/www. who.int/docs/default-source/searo/timor-leste/11-02-2020-tlssitrep-7-ncov-final.pdf?sfvrsn=72720e51_2. Accessed 28/03/2020.

8. Centers for disease control and prevention. Symptoms of Novel Coronavirus (COVID-19). https://www.cdc.gov/ coronavirus/2019-ncov/about/symptoms.html. Accessed 22/03/2020.

9. China's National Health Commission news conference on coronavirus. China's National Health Commission is providing an update on coronavirus outbreak. https://www.aljazeera. com/news/2020/01/chinas-national-health-commission-newsconference-coronavirus-200126105935024.html. Accessed 22/03/2020.

10. Samuel Shen, Ryan Woo. Coronavirus incubation could be as long as 27 days, Chinese provincial government says. The Thomson Reuters Trust Principles. https://www.reuters.com/article/us-china-health-incubation/coronavirus-incubation-couldbe-as-long-as-27-days-chinese-provincial-government-saysidUSKCN20G06W. Accessed 22/03/2020.

11. Yan B, Lingsheng Y, Tao W, Fei T, Dong-Yan J, Lijuan C, et al. Presumed Asymptomatic Carrier Transmission of COVID-19. JAMA 2020; 2(5):23-9.

12. Huang C, Wang Y, Li X, Ren L, Zhao J, Hu Y, et al. Clinical features of patients infected with 2019 novel coronavirus in Wuhan, China. Lancet 2020;395:497-506.

13. Liang W, Guan W, Chen R, Wang W, Li J, Xu K, et al. Cancer patients in SARS-CoV-2 infection: a nationwide analysis in China. Lancet Oncol 2020; 21(3):335-7.

14. Zhou P, Yang XL, Wang XG, Hu B, Zhang L, Zhang W, et al. A pneumonia outbreak associated with a new coronavirus of probable bat origin. Nature 2020;579:270-3.

15. Hamming I, Timens W, Bulthuis ML, Lely AT, Navis G, van Goor H. Tissue distribution of ACE2 protein, the functional receptor for SARS coronavirus. The first step in understanding SARS pathogenesis. J Pathol 2004;203(2):631-37.

16. Wei J, Li Y. Airborne spread of infectious agents in the indoor environment. Am J Inf Control 2016;44(9):102-8. 
17. Cleveland JL, Gray SK, Harte JA, Robison VA, Moorman AC, Gooch BF. Transmission of blood-borne pathogens in US dental healthcare settings: J Am Dental Assoc 2016;147(9):729-38.

18. Li RWK, Leung KWC, Sun FCS. Severe acute respiratory syndrome (SARS) and GDP. Part II: implications for GDPs. Br Dent J 2004;197(3):130-134.

19. Kampf G, Todt D, Pfaender S, Steinmann E. Persistence of coronaviruses on inanimate surfaces and its inactivation with biocidal agents. J Hospital Inf 2020;104(3):246-51.

20. Otter JA. Transmission of SARS and MERS coronaviruses and influenza virus in healthcare settings: the possible role of dry surface contamination. J Hospital Inf 2016;92(3):235-50.

21. Samaranayake LP, Reid J, Evans D. The efficacy of rubber dam isolation in reducing atmospheric bacterial contamination. ASDC J Dentistry Children 1989;56(6):442-4.
22. Samaranayake LP, Peiris M. Severe acute respiratory syndrome and dentistry: a retrospective view. J Am Dental Assoc 2004;135(9):1292-302.

23. Hu T, Li G, Zuo Y, Zhou X. Risk of hepatitis B virus transmission via dental Handpieces and evaluation of an anti-suction device for prevention of transmission. Infect Con Hosp Epidem 2007;28(1):80-2.

24. Sanjay M, Md. IA, Bhoomika A, Ashish G. Use of Newer Protective and Disinfection Strategies: A Simple Tool Guide for the Dentists During the COVID-19 Pandemic. Int J Cur Res Rev 2020;12(16): 321-5. 